(RESEARCH ARTICLE)

\title{
Cancer commonness in Eastern and Western Libya and effect of the eating way on patients
}

\author{
Agila Amal Rajab * \\ Department of Nutrition, Faculty of Medical Technology, Tobruk University, Tobruk, Libya.
}

Publication history: Received on 06 February 2020; revised on 17 February 2020; accepted on 21 February 2020

Article DOI: https://doi.org/10.30574/wjarr.2020.5.2.0035

\begin{abstract}
This work provides a summary on size of food related tumors including pancreas, stomach, liver and colorectal tumors in Eastern and Western Libya from 2005 to 2009; and lung, breast and colorectal cancers at Tobruk Medical Center in 2017, and to recognize the responsibility of bad nutritional habits in growing cancer. A total of 972 cancer cases were analyzed. About, 785 cases (466 males and 319 females) were from Eastern and Western Libya and diagnosed with pancreas, stomach, liver, mouth, pharynx and colorectal cancers. Whereas, 187 cases (40 males and 147 females) were obtained from Tobruk Medical Center and diagnosed with lung, breast and colorectal cancers. A cross sectional study performed on 300 patients with pancreas, stomach, liver, mouth, pharynx and colorectal cancers at Tobruk Medical Center, Al Shefa Clinic and Al Shema Clinic in Tobruk from August 2016 to March 2018. Of 785 patients, there were 382 cases ( 220 males and 162 females) from Western Libya and 403 cases (246 males and 157 females) from Eastern Libya. About $59.4 \%$ males and $40.6 \%$ females were detected with pancreas, stomach, liver, mouth, pharynx and colorectal cancers. Men are just about $18.8 \%$ more expected than women to build up tumors. Approximately $28 \%$ males and $20.6 \%$ females from Western Libya, while 31.4\% males and 20\% females from Eastern Libya. Men in Eastern Libya are almost 3.4\% more likely than men in Western Libya to develop cancer. While, women in Western Libya are only $0.6 \%$ more likely than women in Eastern Libya to develop cancer. Among interviewed patients, $42 \%(\mathrm{n}=126)$ cases commonly consumed fast foods, while $34 \%(\mathrm{n}=102)$ cases rarely ate fruits and vegetables, and $24 \%(\mathrm{n}=72)$ patients had family history. High-quality nutrition with excellent bodily movements encourages health of patients with cancer.
\end{abstract}

Keywords: Food related tumors; Bad nutritional habits; Eastern and western Libya; Cross sectional study; Highquality nutrition.

\section{Introduction}

Cancer reflects on a most significant sickness in Libya [1] and may possibly stand for the second reason of death behind cardiovascular diseases [2]. World Health Organization (WHO) reported that food-related threats may be accountable for some cancer injuries such as colorectal, stomach, liver, pancreas, mouth and pharynx cancers. Colorectal cancer involves cancers of the colon, rectum, appendix and anus. As well, WHO becomes aware of a affiliation between cancer risks and other factors including bad nutritional habits, smoking, and proportion of animal to plant food eaten, value and quality of food, food preparation methods, preserved foodstuff superiority, and standard living style [3]. Worldwide, cancer is quickly becoming a main reason of mortality, especially among aging populations with past family history in the developing countries; also, cancer is becoming a foremost difficulty in Libya. Colorectal cancer represents the second main universal cause of cancer incidence and mortality in both sexes and is an emerging trouble [4]. In Libya, colorectal, liver and stomach cancers are most common and occur due to many risk factors. Living style, non healthy cooking, huge stress, cigarette smoking and cultural nutritional habits may represent cancer risk factors [5]. Cancer incidence in Libya has not been studied for almost a decade [4, 5]. Colorectal, stomach, liver, and pancreas malignancies are the mainly familiar tumor in men. They regard as a significant illness in

\footnotetext{
${ }^{*}$ Corresponding author

E-mail address: amal_agela@yahoo.com
} 
Western and Eastern Libya [5] and may stand for one of main reasons of passing away after cardiovascular diseases. For example, across the world, at least, 151,000 of new colorectal malignancy cases were yearly accounted [6].

Poor diet directs to about $30 \%$ of cancer incidence in developed countries and $20 \%$ in developing countries $[3,6]$. In addition, no diet closely is able to defend from getting cancer, but some natural foodstuffs give a hand promoting body health, maintaining the immune system and reducing the risk of food related cancers [6]. Well nourishment is essential for growth and restores tissues. Consequently, utilization a valuable quantity of fruits, vegetables, fibers, minerals, amino acids, omega-3 and antioxidants may keep a healthy body and diminish cancer danger [6]. Theses natural foodstuffs lend a hand to patients diagnosed with pancreas, stomach, liver, mouth, pharynx; colorectal cancers get the right diet to fight the sickness [7]. According to Better Health Chanel, fruits and vegetables contain plenty of the antioxidants improve the patients diagnosed with the food related cancers [Fact sheet: cancer and food, http://www.betterhealth.vic.gov.au/bhcv2/bhcarticles.nsf/pages/Cancer_and_food. The last accessed on [5/1/2018], especially in the beginning of the disease. The objectives of this research article are firstly; to offer a general idea of size of colorectal, stomach, liver, pancreas, mouth and pharynx cancers incidence in Eastern and Western Libya form 2005 to 2009, and secondly to give information about incidence of lung, breast and colorectal cancers at Tobruk Medical Center in 2017, and finally to spot the role of bad nutritional habits in developing cancer.

\section{Methodology}

\subsection{Data Collection and Analysis}

Data from multiple sources were gathered. Statistics were obtained from Misurata National Cancer Institute Registry, the Department of Oncology at the Benghazi Medical Center, Tripoli Medical Center Registry and Tobruk Medical Center Registry. Data were collected from multiple cities in Eastern and Western Libya including Misurata, Khums, Zliten, Sirt, Tripoli, Mesalata, Jofra, Tarhona, zawea, tobruk, tamimy, derna, beer al ashhab, albeda, benghazi, martoba, shahat, alghaara, kanbot, al berdy, mersalak , and ain mara.

All personal identifiers were stripped from the data and only significant parameters were analyzed. A total of 972 cases (506 males and 466 females) aged from 25 to 80 years were analyzed. Of 972, 785 cases (466 males and 319 females) diagnosed with pancreas, stomach, liver, mouth, pharynx, colorectal cancers during 2005 to 2009. Also, about 187 cases ( 40 males and 147 females) diagnosed with lung, breast and colorectal cancers were obtained from Tobruk Medical Center, Tobruk in 2017 from $1^{\text {th }}$ Jan to $31^{\text {th }}$ Dec, 2017. Data were computerized in a data sheet and percentage formulas of food related cancer patients were calculated using Microsoft office excel 2016 program.

\subsection{Cross Sectional Study}

A self administered questionnaire was conducted on three hundred Libyan patients with pancreas, stomach, liver, mouth, pharynx and colorectal cancers at Tobruk Medical Center, Al Shefa Clinic and Al Shema Clinic in Tobruk during the period from August 2015 to March 2018. The patients aged from 34 to 75 years (132 males and 168 females) and the weight of interviewed patients was from 41 to $94 \mathrm{Kg}$. Data was collected on a form (questionnaire) during the interview with each patient. Participants were asked to detail their nutritional habits throughout one to three years before getting hurt cancer. The questions were only focused on past family history, life style factors including eating red meat, fast foods, drinking gaseous beverages, drinking water and eating fruits and vegetables; and exercise, and smoking status.

\subsection{Statistical Analysis}

An SPSS-based model Statistics Software (version 20.0, SPSS, Inc., Chicago, Illinois, USA) was designed and descriptive statistics were performed. Pearson Chi-square test was used to evaluate the significance of the association between males and females being diagnosed with pancreas, stomach, liver, mouth, pharynx and colorectal cancers. In all tests, $\alpha$ $<0.05$ was regarded statistically significant. All confidence intervals (CIs) were calculated at the $95 \%$ level of statistical significance.

\section{Results and discussion}

\subsection{Data from Misurata, Tripoli and Bengazi Medical Centers (2005 to 2009)}

The significance of this work is that it creates a baseline of incidence of food related cancers which supposed to be the backbone for any future national cancer plan in Libya. The characteristics of pancreas, stomach, liver, mouth, pharynx 
and colorectal cancers population ( $\mathrm{n}=785)$ was observed on many cities in Eastern and Western Libya. Figure (1) gives details about the number of males and females diagnosed with pancreas, stomach, liver, mouth, pharynx and colorectal cancers in Eastern and Western Libya from 2005 to 2009.

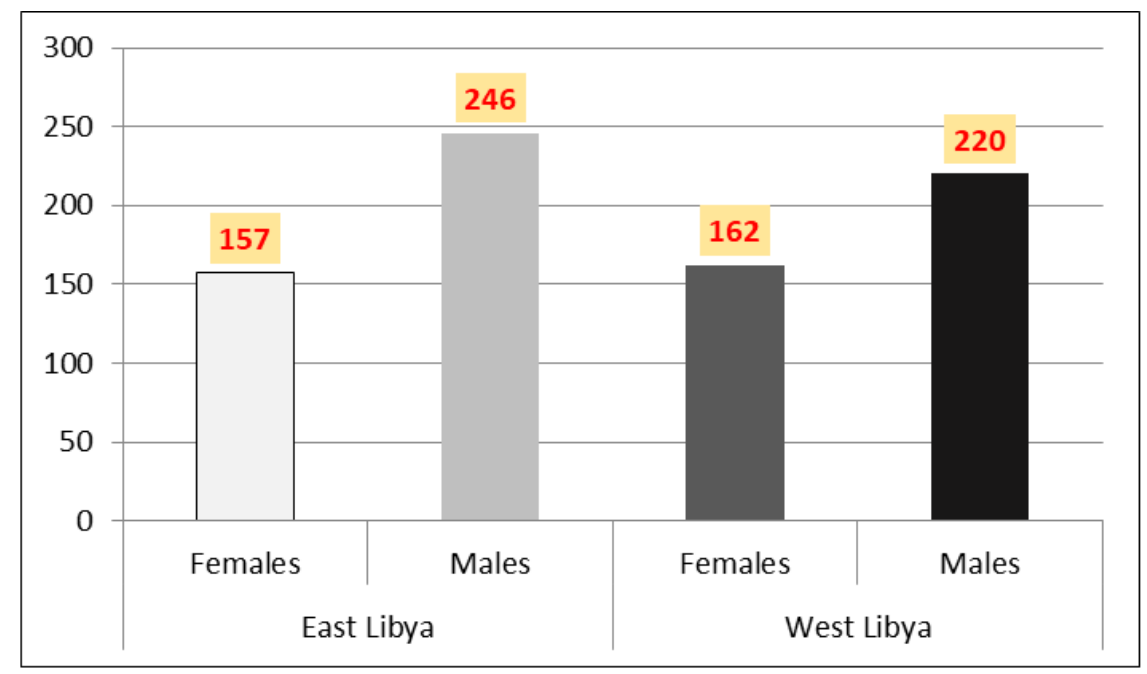

Figure 1 Patients Diagnosed with Pancreas, Stomach, Liver, Mouth, Pharynx and Colorectal Cancers in Eastern and Western Libya from 2005 to 2009 (n=785).

There was no statistically significant association between males and females for getting pancreas, stomach, liver, mouth, pharynx and colorectal cancer $(P=0.319)$. The data gave an idea about pancreas, stomach, liver, mouth, pharynx and colorectal cancers can affect both sexes in Libya. But men are more suspected than women to develop food related cancers. A previous study documented that the general incidence of pancreas, stomach, liver, mouth, pharynx and colorectal cancers was reasonably close between the sexes [5, 6]. Also, another prior study implied that the speed of food related cancers is the maximum in North Africa, where other malignancies happened less frequently [7]. In Canada, patients diagnosed with food related cancers at earlier phases had a more opportunity to survive from the disease than patients diagnosed with food related cancers in North Africa. Because, the reduced quality of medical and nutritional care in North Africa increases the death rates. This gives a clue that the early detection and excellent quality of medical and dietary care represents an essential way to protect from cancer risk and decreases the mortality rate $[6,7]$.

Moreover, among 785 cases (466 males and 319 females), fairly accurately, $59.4 \%$ males and 40.6\% females were detected with pancreas, stomach, liver, mouth, pharynx and colorectal cancers. In this study, one of our significant finding that men are just about $18.8 \%$ more expected than women to build up food related cancers. This is similar to a prior study reported that males were more affected by cancer than females. This may be related to the difference in some cultivating conditions and life style including smoking, living approach, food quality, drinking behaviors and jobrelated exposures [8]. Of 785 cases, there were 382 cases (220 males and 162 females) from Western Libya and 403 cases (246 males and 157 females) from Eastern Libya. Approximately 28\% males and $20.6 \%$ females from Western Libya, whereas 31.4\% males and 20\% females from Eastern Libya. Men in Eastern Libya are almost 3.4\% more likely than men in Western Libya to develop cancer. While, women in Western Libya are only $0.6 \%$ more likely than women in Eastern Libya to develop cancer.

According to Canadian Brest Cancer Foundation, patients diagnosed with food related cancers in North America at earlier steps had a superior possibility to stay alive [Breast cancer in Canada, //www.cbcf.org/Pages/default.aspx, last accessed on [2/3/ 2018]. But, in Libya, the reduced quality of therapeutic and dietary care, lack of a correct documentation system, missing digitalization of patient records and no central authority to go behind cancer patients on a long term basis will increase the cancer incidence and mortality rates [7]. Therefore, understanding the role of the high-quality nutrition with excellent bodily movements may encourage health and physical conditions of patients with pancreas, stomach, liver, mouth, pharynx and colorectal cancers in Libya. With the aim of establish proper cancer control programs, accurate statistics for cancer occurrence are completely crucial [5, 7]. Further scientific studies are required to improve the health based diet with body activities and build outline for a strong medical documentation system for people diagnosed with food related cancers in Libya. Figure (2) shows the total percentage of patients with pancreas, stomach, liver, mouth, pharynx and colorectal cancers in Eastern and Western Libya from 2005 to 2009. 


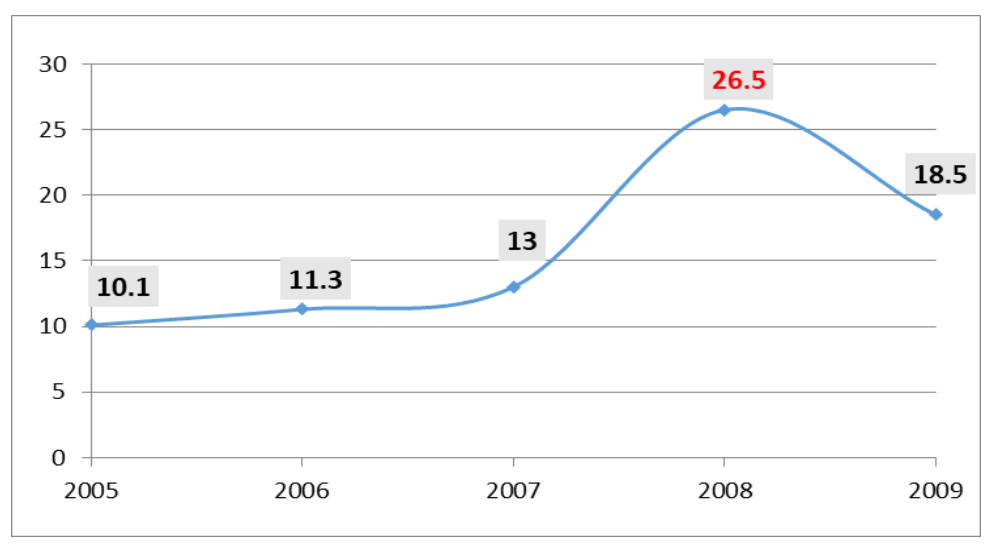

Figure 2 Percentage of Patients Diagnosed with Pancreas, Stomach, Liver, Mouth, Pharynx and Colorectal Cancers in Eastern and Western Libya from 2005 to 2009 ( $\mathrm{n}=785$ ).

One of the essential finding in this effort demonstrated that the percentage of pancreas, stomach, liver, mouth, pharynx and colorectal cancers have been increased from 2005 to 2009 in some Eastern and Western Libya cities, especially in the year 2008. This is similar to a previous study reported that Libya has the maximum rate of food related cancers in North Africa [5, 6, 7] The significant raise in pancreas, stomach, liver, mouth, pharynx and colorectal cancers incidence rates may suggest that there has been no improvement made in cancer control in Libya. This because Libya is missing of national cancer control plans, like to several countries of Eastern Mediterranean Area [8, 9]. Cancer control should diminish the estimated number of new cases and build up the quality of life for those diagnosed with food related cancers [9]. Also, proper control programs necessitate to be in place and healthcare policy should be regulated to take into account the more prevalent and pressing cancers in society. Cancer control requires to be set in place in Libya with the purpose of observe the trends and incidence rates [8,9]. With these founding, we can finally hope to make a change in Libya and raise the level of healthcare facilities.

\subsection{Data Obtained from Tobruk Medical Center in 2017}

Approximately, 187 cases diagnosed with lung, breast and colorectal cancers (40 males and 147 females) were obtained from Tobruk Medical Center, Tobruk from 1th Jan to 31 th Dec, 2017. The results show that about 11 and 29 males diagnosed with lung and colorectal cancers, respectively. While, about 29, 50 and 68 females diagnosed with lung, colorectal and breast cancers, respectively (Figure 3). In this study, lung cancer occurs mostly in females; however, a previous study implied that lung cancer is the foremost cancer in Libya and takes place mostly in smoker men [9].

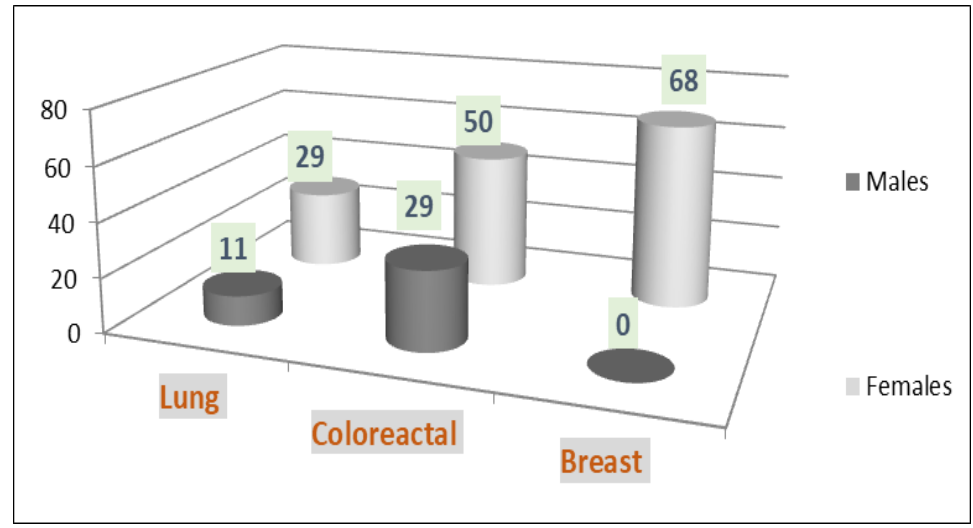

Figure 3 Patients Diagnosed with Lung, Colorectal and Breast Cancers in Tobruk Medical Center in 2017.

Sex-particular incidences were observed substantially between patients diagnosed with cancer. For example, breast cancer occurs only in females and did not occur in female below 20 years old [9]. The higher cases diagnosed with cancer were detected in a specific age period. The most affected age by cancers was in females aged 50 - 55 years followed by the age periods from 45 to 50 and 40 to 45 years, respectively (Figure 4). This explains that progress in the age, especially after 40 years old may affect differently on women to develop breast cancer. This is similar to an earlier study documented that breast cancer is mostly common in women aged more than 40 years $[8,9]$. 


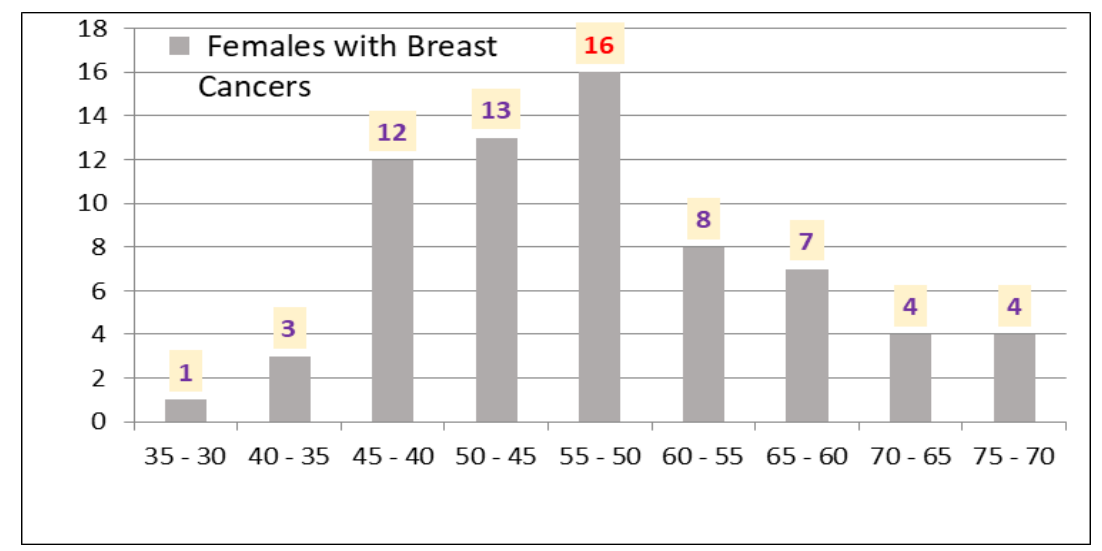

Figure 4 Females Diagnosed with Breast Cancer in Tobruk Medical Center in 2017.

\subsection{Interviewed Patients with Food Related Cancers and Eating Style}

Among 300 interviewed patients diagnosed with pancreas, stomach, liver, mouth, pharynx and colorectal cancers, $42 \%(n=126)$ patients were eating a lot of fast foods, fried potatoes and red meat, while $34 \%(n=102)$ cases rarely ate fruits and vegetables, as well as $24 \%(n=72)$ patients had past family history (Figure 5). Researchers earlier reported that diet may affect on $30 \%$ of all patients diagnosed with cancer $[3,10]$. Moreover, there is a noticeable connection between living style, nutritional habits and cancer incidence [10]. The permanent utilization of red meat (especially fast foods) including beef and lamb, processed meat and saturated fats may enhance the threat of pancreas, stomach, liver, mouth, pharynx and colorectal cancers incidence. However, foods loaded in high-fibers including whole grains, raw vegetables and fruits may defend against cancer because they are physically low in fat and rich in antioxidants and anti-cancer constitutes [10]. Also, this study found that interviewed men were mostly smokers $(90 \%$ $\mathrm{n}=119$ ). A prior study found that $60 \%$ of Libyan males were cigarette smokers $[3,10]$.

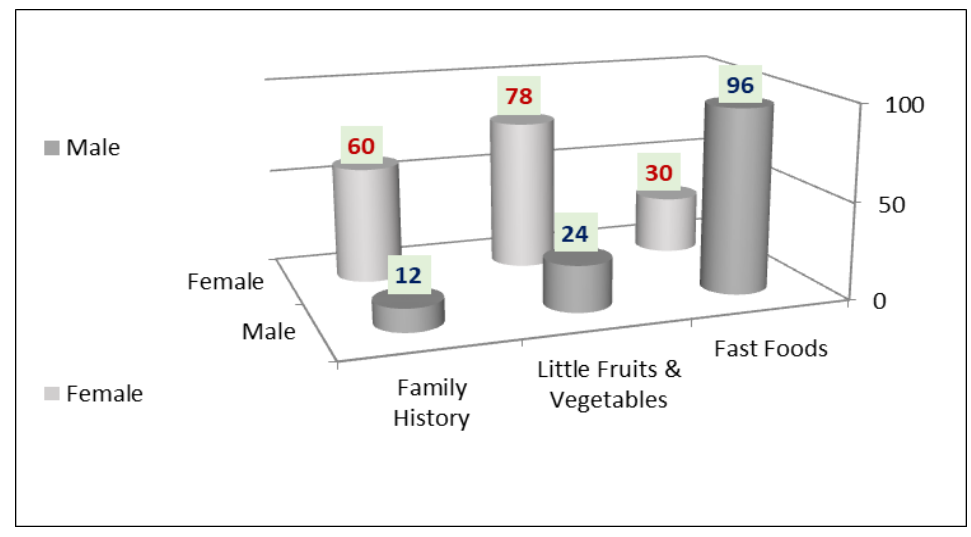

Figure 5 Some Reasons of the Incidence of Pancreas, Stomach, Liver, Mouth, Pharynx and Colorectal cancers ( $\mathrm{n}=300$ ).

\subsection{Nutrition and Food Related Cancers}

The mean factor that can increase the risk of food related cancers is the bad diet. Consequently, the rising incidence of pancreas, stomach, liver, mouth, pharynx and colorectal cancers may be related to alteration in the diet and the standard of living. Also, the role of physical and chemical carcinogens might be taken in hand as a potential risk factor [10]. Scientists direct patients with pancreas, stomach, liver, mouth, pharynx and colorectal cancers to follow prescription instructions in the early stages to protect from the condition [11]. They believe that there is a noticeable connection between living way, dietary patterns and malignancy incidence, because poor nutrition quality may influence on one third of patients diagnosed with cancer. Furthermore, several significant trainings together with doing exercise with a high-quality diet program, stop smoking (especially after meal), avoid alcohol utilization and not skipping breakfast, control weight for obese people are advised and may defend from pancreas, stomach, liver, mouth, pharynx and colorectal cancers [11]. In addition, eating cooking groceries rich in high-fibers (whole grains), drinking 1 to 2 liter of water daily, eating a lot of raw vegetables and fruits without peel, especially carrots, tomatoes, and citrus fruits, apples, grapes and strawberries may protect from food related cancers. Peels of fruits and vegetables may encourage the over growth of normal flora in the intestine and may cause abdomen disorders in food related cancer 
patients. Furthermore, eating cooked vegetables, grains and fish may perhaps defend from cancer injury, as they are visibly low in fat and rich in antioxidants and anti-cancer constitutes [12]. Many scientists encourage using vegetarian diet to protect from cancer.

Conversely, the stable eating of red meats (especially in fast foods) plus eating beef, lamb, processed meats, curing, salting, smoking meats, some sausages and burgers, canned foods, preserved foods, saturated fats, high fat foods, fried foods, margarine, gaseous beverages, and other factors like obesity and smoking may raise the risk of pancreas, stomach, liver, mouth, pharynx and colorectal cancers. Also, smoking represents one of factors of the decline in the developed countries because of health risks $[12,13]$. Patient with food related cancers should have a scheduled nutrition expertise to estimate the diet to keep body weight in a healthy range for height using body mass index measurement (BMI). Body mass index (BMI) represents as obesity index of the individual and was calculated by using the formula BMI = weight in kg's / Height in $\left(\mathrm{m}^{2}\right)$ [13]. Moreover, patient with cancer should drink a valuable amount of water per day and eat more than 5 cups a day varieties of fresh and cooked vegetables (especially, raw vegetables, leafy green vegetables, carrots, tomatoes, broccoli, cabbage and fruits (citrus fruits, in particular) and whole grains. Additionally, patient with food related cancers should eat foods rich in omega-3-fatty acids with sleeping enough hours each night (7-8 hours) and walking for few hours per week with staying away from negative sensations [14].

\section{Conclusion}

The incidence of pancreas, stomach, liver, mouth, pharynx and colorectal malignancies mostly take places in both sexes but it occurs higher in men than women because of the differentiation in some cultural and nutritional habits such as smoking, drinking behaviors and job-related exposures. However, the results from Tobruk Medical Center in the year 2017 indicate that colorectal and lung cancers occur in women than men. The percentage of food related cancer cases have been increased overall during four years in Eastern and Western Libya cities, especially in the year 2008. Men are almost $18.8 \%$ more likely than women to develop pancreas, stomach, liver, mouth, pharynx and colorectal cancers. The interviewed patients had frequently eaten a lot of fast foods, fried potatoes and red meat with eating little of fruits and vegetables before they had cancer, and most of interviewed men were smokers. The most effective age for breast cancer was in women aged from 50 to 55 years.

This study advised that living style and bad nutritional habits including using low quantity of fruits, vegetables and water, eating high calorie fat /meat diet and fast foods; smoking, obesity and huge stress may guide to increase the risks of pancreas, stomach, liver, mouth, pharynx and colorectal cancers. But, permanent consumption of fresh fruits and vegetables without peels, because peels of fruits and vegetables may encourage the over growth of normal flora in the intestine and may cause abdomen disorders. Also, eating cooked vegetables, whole grains, healthy fat containing foods such as avocado, olive oil and nuts may prevent the harm of pancreas, stomach, liver, mouth, pharynx and colorectal cancers. Further research needed for improving cancer awareness and promoting early detection to minimize the risk of pancreas, stomach, liver, mouth, pharynx and colorectal cancers. Our finding suggests that the lack of knowledge about food related cancer is an important factor affect cancer incidence in Libya, thus there is a need for public educational and nutritional programs about cancer and diet, especially for less educated people.

\section{Compliance with ethical standards}

\section{Acknowledgments}

We acknowledges the Tripoli, Benghazi, and Tobtuk Medical Centers, National Cancer Institute in Misurata , Al Shefa clinic and Al Shema Clinic in Tobruk as well as all the participants in this work for providing the research facilities.

\section{Statement of ethical approval}

This study is a part of food related cancer studies. The study protocol was approved by the ethics committee of the Scientific Research in Tobruk University. All patients with pancreas, stomach, liver, mouth, pharynx and colorectal cancers were informed about the research aim and gave oral consent. No patient refused our aim for this study.

\section{References}

[1] El Mistiri M, Pirani M, El Sahli N, El Mangoush M, Attia A, Shembesh R, Habel S, El Homry F, Hamad S and Federico M. (2010). Cancer profile in Eastern Libya: incidence and mortality in the year 2004. Annals of Oncology, 21(9), $1924-1930$. 
[2] Singh R and Al-Sudani OE. (2001). Cancer mortality in Benghazi, Libyan Arab Jamahiriya, 1991-96. Eastern Mediterranean Health Journal, 7(1-2), 255 - 273.

[3] Tantamango-Bartley Y, Jaceldo-Siegl K, Fan J and Fraser G. (2013). Vegetarian diets and the incidence of cancer in a low-risk population. Cancer Epidemiology Biomarker Preview, 22(2), 286 - 294.

[4] El Mistiri M, Verdecchia A, Rashid I, El Sahli N, El Mangush M and Federico M. (2007). Cancer incidence in eastern Libya: The first report from the Benghazi Cancer Registry, 2003. Epidemiology, 120(2), 392-397.

[5] Bodalal Z and Bendardaf R. (2014). Colorectal carcinoma in a Southern Mediterranean country: the Libyan scenario. World Journal Gastrointestinal Oncology, 6 (4), 98-103.

[6] Elzouki AY, Buhjab SI, Alkialani A, Habel S and Sasco AJ. (2012). Gastric cancer and Helicobacter Pylori infection in the Eastern Libya: A descriptive epidemiological study. Arab Journal of Gastroenterology, 13(2), 85-88.

[7] Ermiah EA, Abdalla FN, Buhmeida A, Larbesh ED, Pyrhonen SN and Collan Y. (2012). Diagnosis delay in Libyan female breast cancer. BMC Research Notes, 5(1), 452.

[8] McCann J. (2000). Gender differences in cancer that don't Make sense-or do they? Journal of the National Cancer Institute, 92(19), 1560-1562.

[9] Abusaa A. (2006). Sabratha cancer registry: first annual report, population based cancer registry. African Oncology Institute, Sabratha, Libya, 1 - 60.

[10] Philips R. (1975). Role of life-style and dietary habits in risk of cancer among seventh-day adventists. Cancer Research, 35(11 Pt. 2), 3513 - 3522.

[11] Ghalaita AAB, Shanbih FMH, Hussain MA and Rajan AV. (2014). Enhancing healthy lifestyle of UAE nationals in Dubai. Discovery, 25(90), 75-82.

[12] Frentzel-Beyme R and Chang Claude J. (1994). Vegetarian diet and colon cancer: the German experience. American Journal of Clinical Nutrition, 59(5 Suppl), 1143S-1152S.

[13] Kusumaneela BK, Santhi KV, Afnan SK, Krishna VP and Kusuma MN. (2015). Effect of diet counseling on type 2 diabetes mellitus. International Journal Science Technology, 8, 112 - 118.

[14] Gathani T, Barnes I, Ali R, Arumugham R, Chacko R, Digumarti R, Jivarajani P, Kannan R, Loknatha D, Molhotra $\mathrm{H}$ and Mathew BS. (2017). Lifelong vegetarianism and breast cancer risk: a large multicentre case control study in India. BMC Women's Health, 17, 6.

\section{How to cite this article}

Agila AR. (2020). Cancer commonness in Eastern and Western Libya and effect of the eating way on patients. World Journal of Advanced Research and Reviews, 5(2), 186-192. 